\title{
List of reviewers, 2016
}

Peer review is the life blood of this Journal. The Editors are deeply grateful to the following experts who freely gave their time during 2016 to review articles submitted for publication.

Andrew Argent

Debbie Baker

Janet Bell

Petra Brysiewicz

Kim de Vasconcellos

Roger Dickerson

Konstantin Dimitriades

Rencia Gillespie

Indiran Govender

Susan Hanekom

Andre Heyns

Lauren Hill

Peter Hodkinson

Anri Human

Portia Jordaan

Ivan Joubert

\author{
Robert Kleinloog \\ Heide Kunzmann \\ David Linton \\ Alison Lupton-Smith \\ Mervyn Mer \\ Malcolm Miller \\ Gladness Nethathe \\ Sam Ntuli \\ Natasha Plani \\ Richard Raine \\ Ronel Roos \\ Shelley Schmollgruber \\ David Thompson \\ Mariane van der Heever \\ Monique van Dyk \\ Norbert Welcowics
}

Critical Care Society of Southern Africa

Contact details:

Alison Shaw, CCSSA Secretariat,

5 Hayman Park, Sunbury Park, Douglas Saunders Drive, La Lucia Ridge,

KwaZulu-Natal, 4051, South Africa

Tel: (031) 8316416 ,

Email: secretariat@criticalcare.org.za, Website: www.criticalcare.org.za 